\title{
Supplementary Appendix for Tradeoffs in Introduction Policies for the Anti-Tuberculosis Drug Bedaquiline: A Model Based Analysis
}

\author{
Amber Kunkel, Frank G. Cobelens, Ted Cohen
}

\section{Contents}

1. Summary of included studies involving bedaquiline (p.1)

2. Additional calculation details (p.3)

3. Value of Information analysis (p.7)

4. Sensitivity to distributional assumptions (p.7)

5. Mortality sensitivity analysis, rapid second-line DSTs (p.8)

6. Additional analyses of XDR plus bedaquiline resistance (p.9)

7. Table of fixed parameters (p.10)

8. Complete list of model transitions (p.16)

9. References (p.25)

\section{SUMMARY OF INCLUDED STUDIES INVOLVING BEDAQUILINE}

The following list describes the clinical trial and cohort data on bedaquiline safety and efficacy that were used to inform this analysis. To our knowledge, this was at the time of our analysis an exhaustive list of the published data available on patients receiving bedaquiline for a full 6 months.

1

Title: Multidrug-resistant tuberculosis and culture conversion with bedaquiline

Authors: Diacon AH, Pym A, Grobusch MP, de los Rios JM, Gotuzzo E, Vasilyeva I et al.

Setting: Brazil, India, Latvia, Peru, the Philippines, Russia, South Africa, and Thailand

Study Design: Placebo-controlled clinical trial (bedaquiline or placebo added to optimized background regimen)

\section{Number of Patients:}

With 6 Months of Follow-Up: 66 (Modified Intention-to-Treat, assigned to bedaquiline)

With 24 Months of Follow-Up: 66 (Modified Intention-to-Treat, assigned to bedaquiline)

\section{Overview:}

As a placebo-controlled clinical trial, this study represents the highest quality of evidence available so far. Bedaquiline was found to reduce the median time to culture conversion to 83 days, compared with 125 days with placebo. Ten patients (of 79) in the bedaquiline group died during follow-up, compared with 2 (of $81)$ in the placebo group $(\mathrm{P}=0.02)$. None of the deaths were thought to be related to the study drugs, based on the judgment of a blinded investigator.

2

Title: Bedaquiline in the treatment of multidrug- and extensively drug-resistant tuberculosis 
Authors: Pym AS, Diacon AH, Tang SJ, Conradie F, Danilovits M, Chuchottaworn C et al.

Setting: China, South Korea, the Philippines, Thailand, Estonia, Latvia, Russia, Turkey, Ukraine, Peru, and South Africa

Study Design: Open-label, single-arm trial

\section{Number of Patients:}

With 6 Months of Follow-Up: 205 (Modified Intention-to-Treat group)

With 24 Months of Follow-Up: 205 (Modified Intention-to-Treat group)

\section{Overview:}

This study contains the largest published cohort of patients receiving treatment with bedaquiline available at the time of our analysis. Patients were followed through 120 weeks. 16 deaths $(6.9 \%$ of 233 patients, a lower proportion than that observed in Diacon et al) occurred during follow-up. The median time to culture conversion was 84 days. Although these results appear to represent an improvement over historical MDR TB cohorts, the trial setting and lack of a control group may limit the generalizability of these findings.

\section{3}

Title: Compassionate use of bedaquiline for the treatment of multidrug-resistant and extensively drugresistant tuberculosis: interim analysis of a French cohort

Authors: Guglielmetti L, Le D D, Jachym M, Henry B, Martin D, Caumes E et al.

Setting: France

Study Design: Cohort of patients receiving bedaquiline

\section{Number of Patients:}

With 6 Months of Follow-Up: 35

With 24 Months of Follow-Up: None reported

\section{Overview:}

This study provides one of the earliest looks at bedaquiline used under programmatic conditions. The observed culture conversion rate was $97 \%$ after 6 months of therapy. Bedaquiline was frequently combined with linezolid and either a fluoroquinolone or second-line injectable. The results of this study are limited by its short duration of follow-up and lack of a control arm.

4

Title: Treatment of drug-resistant tuberculosis with bedaquiline in a high HIV prevalence setting: an interim cohort analysis

Authors: Ndjeka N, Conradie F, Schnippel K, Hughes J, Bantubani N, Ferreira H et al.

Setting: South Africa

Study Design: Cohort of patients receiving bedaquiline

\section{Number of Patients:}

With 6 Months of Follow-Up: 63

With 24 Months of Follow-Up: None reported

\section{Overview:}

This study contains the earliest published data on programmatic use of bedaquiline in a setting with high HIV prevalence. $76 \%$ of patients who completed six months of follow up either achieved culture conversion or remained culture negative. The authors highlighted the contrast between these results and those of the 
2012 South Africa NTP MDR-TB cohort, in which culture conversion was only $56 \%$ at 6 months, but noted that other factors, such as expanded availability of Xpert MTB/RIF and linezolid, could explain some of the observed improvements. The results of this study, as for Guglielmetti et al., are limited by its short duration of follow-up and lack of a control arm.

5

Title: New developments in the treatment of drug-resistant tuberculosis: clinical utility of bedaquiline and delamanid (referencing presentation entitled: Armenian experience on treatment of XDR and pre-XDR patients with new drugs under compassionate use program)

Authors: Brigden G, Hewison C, Varaine F (referencing presentation by Yeghiazaryan L).

Setting: Armenia

Study Design: Cohort of patients receiving bedaquiline

\section{Number of Patients:}

With 6 Months of Follow-Up: 32

With 24 Months of Follow-Up: None reported

\section{Overview:}

This review paper included data presented at the 2015 Tuberculosis Symposium Eastern Europe and Central Asia on the Armenian experience on treatment of XDR and pre-XDR patients with new drugs under compassionate use program (available at: http://www.tb-symposium.org/2015/documents/en/presentations/ Lusine_Yeghiazaryan_BDQ_practical_use_eng.pdf). $84 \%$ of patients who were culture positive at the time of treatment initiation had converted by 6 months. This study also had short follow-up and no control arm.

\section{ADDITIONAL CALCULATION DETAILS}

\section{General Calculation Principles}

\section{Accounting for conditional probabilities}

In constructing the model, the potential events for each week were modeled in sequence. For each weekly cycle we gave priority to events in an order that reflected the way they would be recorded as treatment outcomes. For example, for people receiving treatment we first we recorded all deaths. Those people who did not die could end treatment routinely, or if not they could end treatment prematurely (default). We assumed only patients who remained on treatment could have culture converted, and only those who did not culture convert could have acquired resistance (to at most one drug per week). To account for ordering, we input the probability of each event conditional on not experiencing any of the events earlier in the calculation sequence that week.

Examples:

- Weekly probability of culture conversion - input conditional on not dying, defaulting, or finishing treatment that week

- Weekly probability of stopping treatment routinely - conditional on not dying that week

- Weekly probability of default - conditional on not dying or stopping treatment routinely that week 


\section{Converting rates to weekly probabilities}

To convert rates to weekly probabilities $(p)$, we first converted them to rates per week. We then used the following formula:

$$
p=1-\exp (- \text { rate })
$$

Examples:

- Added mortality BDQ (rate 5 per 100 person-years $\rightarrow$ rate 5 per 5200 person-weeks $=0.00096$ per person-week $\rightarrow$ weekly probability $1-\exp (-0.00096)=0.00096)$

\section{Resistance and relapse probabilities}

To convert the probability of acquiring resistance prior to death, conversion, default, or stopping treatment $(Q)$ into weekly probabilities, we used the following equation with the weekly probabilities of each event $(p)$ :

$$
Q_{\text {res }}=\frac{p_{\text {res }}}{p_{\text {die }}+p_{\text {default }}+p_{\text {stop }}+p_{\text {convert }}+p_{\text {res }}}
$$

Examples:

- Probability acquired BDQ resistance given XDR

- Probability acquired XDR given pre-XDR, not on BDQ

- All other acquired resistance parameters (but may include additional considerations below)

A similar equation was used to relate the weekly cure rate to the probability of moving from "culture negative" (high risk of relapse immediately after treatment) to "stable cure" prior to death, default, or stopping treatment.

\section{Median time to culture conversion}

Based on our literature review, we estimated the median time to culture conversion (if no one had died or stopped treatment) to be approximately 13 weeks for people initially MDR, 18 weeks for people initially pre-XDR, and 26 weeks for people initially XDR. We used TreeAge to estimate a weekly probability of conversion based on these targets and our fixed weekly probabilities of acquiring pre-XDR and XDR TB.

To simplify our sensitivity analyses, we chose to consider the effect of BDQ on the median time to culture conversion if no one had died, stopped treatment, or acquired resistance. We did this by calculating the weekly probabilities of conversion from above to the median time of culture conversion if no one had died, stopped treatment, or acquired resistance. Our bedaquiline multiplier was then applied to these values.

We converted median time to event parameters (in weeks) to weekly probabilities using the geometric distribution:

$$
P=1-2^{-1 / M}
$$

\section{Probability acquiring pre-XDR without BDQ}

From the literature, we estimated that the probability of acquiring resistance to any fluoroquinolone given initial MDR was approximately 0.065 , and that the probability of acquiring resistance to any second-line 
injectable was similar. We also determined that the risk of XDR given pre-XDR was approximately 0.26. Note the informal notation: $P(\operatorname{PreX} D R \mid M D R)$ is the probability of developing at least PreXDR for an individual who is initially MDR, and not receiving bedaquiline.

$$
\begin{gathered}
P(\operatorname{PreX} D R \mid M D R)=P(F Q R \mid M D R)+P(2 L I R \mid M D R)-P(X D R \mid M D R) \\
P(\operatorname{PreX} D R \mid M D R)=P(F Q R \mid M D R)+P(2 L I R \mid M D R)-P(\operatorname{PreX} D R \mid M D R) P(X D R \mid \text { PreXDR }) \\
P(\operatorname{PreX} D R \mid M D R)=.065 * 2-0.26 * P(\operatorname{PreX} D R \mid M D R) \\
P(\operatorname{PreX} D R \mid M D R)=0.103
\end{gathered}
$$

\section{Probability acquiring pre-XDR with BDQ}

This is similar to the section above, except we also need to account for the potential to acquire resistance to bedaquiline. Note the informal notation: $\operatorname{P}(\operatorname{PreX} D R \mid M D R)$ is the probability of developing at least PreXDR for an individual who is initially MDR (and BDQ sensitive), on bedaquiline.

$$
\begin{gathered}
P(\operatorname{Pre} X D R \mid M D R)=P(F Q R \mid M D R)+P(2 L I R \mid M D R)-P(X D R \mid M D R) \\
P(\operatorname{PreX} D R \mid M D R)=P(F Q R \mid M D R)+P(2 L I R \mid M D R)-P(\text { PreXDR } \mid M D R) P(X D R \mid \text { develop PreXDR })
\end{gathered}
$$

The issue here is that $P(X D R \mid$ develop Pre $X D R)$ depends on whether the individual already has resistance to BDQ at the time they become PreXDR or not.

$$
\begin{aligned}
& P(X D R \mid \text { develop PreXDR })=P(\text { already BDQR develop PreX } D R) P(X D R \mid \text { PreX } D R, B D Q R) \\
& +(1-P(\text { already } B D Q R \mid \text { develop PreX } D R)) P(X D R \mid \text { PreX } D R, B D Q S)
\end{aligned}
$$

To solve for $P($ already $B D Q R \mid$ develop PreXDR), we can use our knowledge of the risk of resistance to bedaquiline for people who are initially MDR vs preXDR.

$$
\begin{aligned}
& P(B D Q R \mid M D R)=P(B D Q R \text { prior to/without PreX } D R) \\
& +(1-P(\text { already } B D Q R \mid \text { develop PreX } D R)) P(\text { PreX } D R \mid B D Q S, M D R) P(B D Q R \mid \text { PreXDR, BDQS })
\end{aligned}
$$

$P($ already BDQR $\mid$ develop PreX $D R)=\frac{P(B D Q R \text { prior to } / \text { without PreX } D R) P(\operatorname{PreX} D R \mid M D R, B D Q R)}{P(\operatorname{PreX} D R \mid M D R)}$

Let $\mathrm{X}$ be the probability of developing bedaquiline resistance either prior to or without developing preXDR for people initially MDR on BDQ. Let Y be the probability of ever developing pre-XDR for people initially MDR on BDQ.

Let $Q_{f m}$ be the probability of ever developing FQ resistance given MDR on BDQ. Let $Q_{x p b}$ be the probability of ever developing XDR given pre-XDR and BDQ resistance. Let $Q_{x p}$ be the probability of ever developing XDR given pre-XDR and BDQ sensitivity. Let $Q_{b m}$ be the probability of developing bedaquiline resistance given initially $\mathrm{MDR}$ and $\mathrm{BDQ}$ sensitive. Let $Q_{b p}$ be the probability of developing bedaquiline resistance given initially pre-XDR and BDQ sensitive. Let $Q_{p m b}$ be the probability of developing pre-XDR given initially MDR and bedaquiline resistant.

$$
Y=2 Q_{f m}-Y\left(\frac{X Q_{x p b} Q_{p m b}}{Y}+\left(1-\frac{X Q_{p m b}}{Y}\right) Q_{x p}\right)
$$




$$
Q_{b m}=X+\left(1-\frac{X Q_{p m b}}{Y}\right) Y Q_{b p}
$$

Solving the first equation for X:

$$
\begin{gathered}
Q_{b m}-Y Q_{b p}=X-X Q_{p m b} Q_{b p} \\
Q_{b m}-Y Q_{b p}=X\left(1-Q_{p m b} Q_{b p}\right) \\
\frac{Q_{b m}-Y Q_{b p}}{1-Q_{p m b} Q_{b p}}=X
\end{gathered}
$$

Substituting into the first equation:

$$
\begin{gathered}
Y=2 Q_{f m}-Y\left(\frac{X Q_{x p b} Q_{p m b}}{Y}+\left(1-\frac{X Q_{p m b}}{Y}\right) Q_{x p}\right) \\
Y=2 Q_{f m}-X Q_{x p b} Q_{p m b}-Y Q_{x p}+X Q_{x p} Q_{p m b} \\
Y=2 Q_{f m}-X Q_{p m b}\left(Q_{x p b}-Q_{x p}\right)-Y Q_{x p} \\
Y=2 Q_{f m}-\frac{Q_{b m}-Y Q_{b p}}{1-Q_{p m b} Q_{b p}} Q_{p m b}\left(Q_{x p b}-Q_{x p}\right)-Y Q_{x p}
\end{gathered}
$$

We used Matlab's symbolic toolbox to solve this equation for Y. We checked this equation by verifying that our TreeAge model gave similar results for $\mathrm{X}$ and the probability of developing XDR given initially MDR and BDQ sensitive for a typical parameter set.

\section{Probability acquiring XDR or BDQ resistance - from pre-XDR receiving BDQ}

People can develop XDR without or prior to developing BDQ resistance, or they can acquire BDQ resistance first, increasing their chances of developing XDR. Similarly people can develop BDQ resistance without, prior to, or after developing XDR.

Let $\mathrm{X}$ be the weekly probability of developing $\mathrm{BDQ}$ resistance given pre-XDR. Let $\mathrm{Y}$ be the weekly probability of developing XDR given pre-XDR. Let A be the weekly probability of any other possible event (probability of dying or defaulting or finishing treatment or culture converting).

Let $Q_{b p}$ be the probability of ever developing BDQ resistance starting pre-XDR, on BDQ. Let $Q_{x p}$ be the probability of ever developing XDR starting pre-XDR, BDQ-sensitive, on BDQ. Let $Q_{b x}$ be the probability of ever developing BDQ resistance starting XDR. Let $Q_{x p b}$ be the proportion of people with pre-XDR and BDQ resistance who acquire XDR prior to death, default, finishing treatment, or culture conversion.

We can then use the following equations to solve for $X$ and $Y$.

$$
\begin{aligned}
Q_{b p} & =\frac{X}{X+Y+A}+Q_{b x} \frac{Y}{X+Y+A} \\
Q_{x p} & =\frac{Y}{X+Y+A}+Q_{x b p} \frac{X}{X+Y+A}
\end{aligned}
$$

Therefore

$$
\begin{gathered}
Q_{b p}(X+Y+A)=X+Q_{b x} Y \\
Q_{x p}(X+Y+A)=Y+Q_{x b p} X
\end{gathered}
$$

Solving for X first: 


$$
\begin{gathered}
\left.\left(Q_{b p}\right)-1\right) X=Q_{b x} Y-Q_{b p}(Y+A) \\
X=\frac{\left(Q_{b x}-Q_{b p}\right) Y-Q_{b p} A}{Q_{b p}-1}
\end{gathered}
$$

Plugging into the equation for Y:

$$
\begin{gathered}
Q_{x p}(X+Y+A)=Y+Q_{x b p} X \\
\left(Q_{x p}-Q_{x b p}\right) X+Q_{x p} A=Y-Q_{x p} Y \\
\left(Q_{x p}-Q_{x b p}\right)\left(\frac{\left(Q_{b x}-Q_{b p}\right) Y-Q_{b p} A}{Q_{b p}-1}\right)+Q_{x p} A=Y-Q_{x p} Y \\
Q_{x p} A-\left(Q_{x p}-Q_{x b p}\right)\left(\frac{Q_{b p} A}{Q_{b p}-1}\right)=Y-Q_{x p} Y-\left(Q_{x p}-Q_{x b p}\right)\left(\frac{\left(Q_{b x}-Q_{b p}\right) Y}{Q_{b p}-1}\right) \\
Q_{x p} A-\frac{\left(Q_{x p}-Q_{x b p}\right) Q_{b p} A}{Q_{b p}-1}=Y\left(1-Q_{x p}-\frac{\left(Q_{x p}-Q_{x b p}\right)\left(Q_{b x}-Q_{b p}\right)}{Q_{b p}-1}\right) \\
Y=\frac{Q_{x p} A\left(Q_{b p}-1\right)-\left(Q_{x p}-Q_{x b p}\right) Q_{b p} A}{\left(Q_{b p}-1\right)\left(1-Q_{x p}\right)-\left(Q_{x p}-Q_{x b p}\right)\left(Q_{b x}-Q_{b p}\right)}
\end{gathered}
$$

\section{VALUE OF INFORMATION ANALYSIS}

We additionally conducted a Value of Information analysis to support our assessment of the parameters with greatest influence on our life expectancy outcomes (see Figures 3 and 4 in the Main Text). We first calculated the expected value of perfect information, or the additional life expectancy that one would expect to gain on average if there was no uncertainty in our bedaquiline-related parameters, based on 5,000 random parameter samples. We then calculated the expected value of partial perfect information separately for each of the bedaquiline parameters, sampling 200 values in the outer loop of the parameter of interest and 1000 values in the inner loop of the remaining parameters.

The results of our value of information analysis are provided in Table 1 . The expected value of perfect information for all parameters is 0153 life years (per patient). The expected value of partial perfect information was 0 for all parameters except the ratio of median time to culture conversion on vs. off bedaquiline (0074) and the bedaquiline-associated mortality rate (0006). These results indicate that uncertainty in the optimal use of bedaquiline to improve life expectancy reflects primarily assumptions about efficacy and (possible) drug-related mortality, rather than resistance. These results are supported by the Main Text Figure 4, in which providing bedaquiline to all patients was most frequently the optimal strategy across all values of the parameters excluding culture conversion and mortality. Although their interpretation is limited to our assumed parameter distributions, they overall support the conclusion that understanding these parameters should be emphasized in future studies of bedaquiline.

\section{SENSITIVITY TO DISTRIBUTIONAL ASSUMPTIONS}

To assess the sensitivity of our results to the assumed uniform distributions, we reran the life expectancy portion of the model assuming triangular distributions with the same limits as in the Main Text Table 1 and greatest density at the midpoint. The results are shown here in Appendix Table 4. Although the simulation mean values of average life expectancy are similar to those produced by our initial uniform distributions, the bounds around these estimates are tighter, reflecting the shape of the applied triangular distributions. Furthermore, use of this distribution results in the "All MDR" strategy being optimal for a greater proportion 
Table 1: Value of Information analysis results

\begin{tabular}{|l|l|}
\hline \multicolumn{2}{|c|}{ Expected Value of Perfect Information } \\
\hline Overall & 0.153 \\
\hline Expected Value of Partial Perfect Information (BDQ Parameters) \\
\hline Default rate on BDQ & 0 \\
Relapse risk on BDQ & 0 \\
Time to culture conversion on BDQ & 0.074 \\
BDQ-related mortality & 0.006 \\
Protection from resistance to OBR & 0 \\
Rate of resistance to BDQ & 0 \\
\hline
\end{tabular}

Table 2: Life expectancy results using triangular distributions with greatest density at the midpoint. Results for average life expectancy are given as simulation mean (2.5 percentile, 97.5 percentile). Results for percent of simulations optimal are calculated out of 5,000.

\begin{tabular}{l|llll} 
& All MDR & PreXDR Only & XDR Only & None \\
\hline Average Life Expectancy & $36.0(34.2,37.9)$ & $35.1(34.6,35.6)$ & $34.9(34.7,35.1)$ & 34.8 \\
Percent of Simulations Optimal & 87.2 & 0.0 & 0.0 & 12.7
\end{tabular}

of simulations than obtained under our initial distributions, though the "None" strategy remains optimal for $12.7 \%$ of simulations.

\section{MORTALITY SENSITIVITY ANALYSIS, RAPID SECOND-LINE DSTs}

Figure 1 shows results similar to Figure 4 in the main text, except assuming the availability of rapid second-line DSTs. In this scenario, the intermediate "PreXDR+XDR" bedaquiline is more frequently preferred than when rapid second-line DSTs are unavailable.

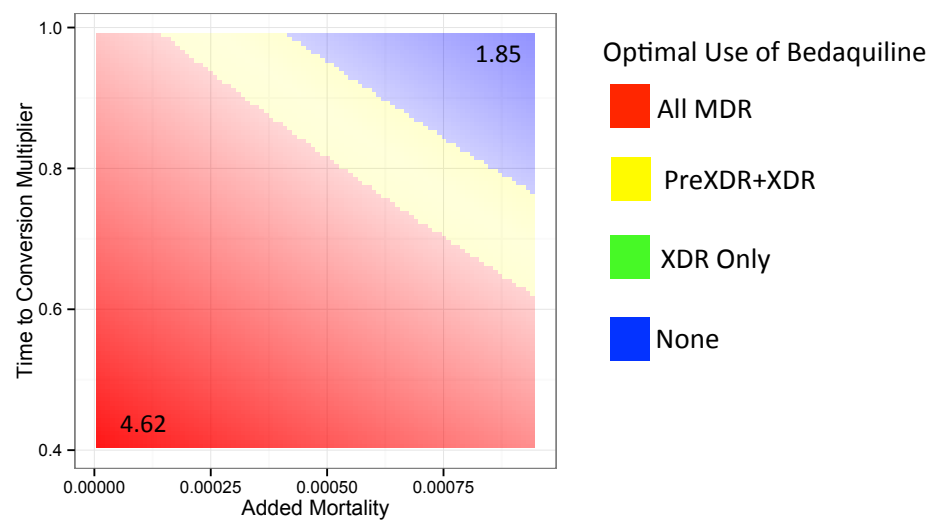

Figure 1: Heat maps showing regions in which each bedaquiline use strategy would be preferred if rapid second-line DSTs were available. The $\mathrm{x}$ and $\mathrm{y}$ axes show the explored rates of (relative) median time to culture conversion and added mortality associated with bedaquiline use. Remaining parameters are fixed at their midpoints. Colors indicate the optimal bedaquiline use strategy, and shading indicates the magnitude of difference in average life expectancy between the best and worst strategies, with the corner values listed on the figure. 


\section{ADDITIONAL ANALYSES OF XDR PLUS BEDAQUILINE RESISTANCE}

To greater understand the results for our "acquired XDR plus resistance to bedaquiline" outcome, we repeated the analyses under the assumption that rapid second-line DSTs were available, as well as for different distributions of baseline resistance patterns. The results show that if rapid second-line DSTs were available, the preferred strategy would most frequently be to provide bedaquiline to all patients. Providing bedaquiline to all patients is also the most frequently preferred strategy under our baseline DST scenario when all patients are initially MDR or PreXDR. However, when all patients are initially XDR, providing bedaquiline to all patients with MDR TB is never the preferred strategy. In this case, the additional delays in starting bedaquiline prevent some patients from receiving bedaquiline (and being at risk of acquired drug resistance) prior to dying, stopping treatment, or converting to culture negative.

Table 3: Percentage of simulations for which each bedaquiline use policy minimizes acquired XDR plus resistance to bedaquiline, comparing our baseline to scenario to a rapid second-line DST scenario and different baseline resistance distributions.

\begin{tabular}{l|lllll} 
& \multicolumn{5}{|c}{ Percentage of Simulations Minimizing XDR+BDQR } \\
BDQ Available For & Baseline Results & Rapid DST & All Initially MDR & All Initially PreXDR & All Initially XDR \\
\hline XDR Only & 10.8 & 0.0 & 4.0 & 10.5 & 100.0 \\
PreXDR+XDR & 85.6 & 1.5 & 0.0 & 0.0 & NA (same as XDR) \\
All MDR & 3.6 & 98.5 & 96.0 & 89.5 & 0.0
\end{tabular}

Table 4: Percentage of patients acquiring XDR plus resistance to bedaquiline, comparing our baseline to scenario to a rapid second-line DST scenario and different baseline resistance distributions. Results are given as are given as simulation mean (2.5 percentile, 97.5 percentile).

\begin{tabular}{l|lllll} 
& \multicolumn{5}{|c}{ Percentage of Patients Acquiring XDR+BDQR } \\
BDQ Available For & Baseline Results & Rapid DST & All Initially MDR & All Initially PreXDR & All Initially XDR \\
\hline XDR Only & $3.5(1.7,5.6)$ & $5.3(2.0,8.4)$ & $0.7(0.3,1.1)$ & $6.2(2.3,9.9)$ & $21.2(7.9,34.0)$ \\
PreXDR+XDR & $3.2(1.2,5.2)$ & $3.7(1.4,6.4)$ & $0.6(0.2,1.0)$ & $5.2(2.0,9.2)$ & $21.2(7.9,34.0)$ \\
All MDR & $3.4(1.3,6.2)$ & $3.4(1.3,6.2)$ & $0.2(0.1,0.9)$ & $4.0(1.4,9.6)$ & $33.5(12.6,53.2)$
\end{tabular}


FIXED PARAMETERS

\begin{tabular}{|c|c|c|c|c|}
\hline Name & Description & Calculation \& Units & Value & Explanation \\
\hline Age & Age starting treatment & Years & 30 & \\
\hline InitMDR & $\begin{array}{l}\text { Proportion of people } \\
\text { initiating MDR } \\
\text { treatment without pre- } \\
\text { XDR or XDR TB }\end{array}$ & Proportion & 0.671 & {$[1]$} \\
\hline InitPreXDR & $\begin{array}{l}\text { Proportion of people } \\
\text { initiating MDR } \\
\text { treatment with pre- } \\
\text { XDR TB }\end{array}$ & Proportion & 0.262 & {$[1]$} \\
\hline InitXDR & $\begin{array}{l}\text { Proportion of people } \\
\text { initiating MDR } \\
\text { treatment with XDR } \\
\text { TB }\end{array}$ & Proportion & 0.067 & {$[1]$} \\
\hline MedianCCMDR & $\begin{array}{l}\text { Median time to culture } \\
\text { conversion - MDR, } \\
\text { not on BDQ }\end{array}$ & $\begin{array}{l}\text { median time in weeks, assuming no death, } \\
\text { default, or acquired resistance }\end{array}$ & $\begin{array}{l}12.82 \text { (13 allowing } \\
\text { for acquired } \\
\text { resistance) }\end{array}$ & $\begin{array}{l}\text { [2] (13 weeks for all } \\
\text { patients), [3] (13 weeks for } \\
\text { MDR only), [4] }\end{array}$ \\
\hline MedianCCPreXDR & $\begin{array}{l}\text { Median time to culture } \\
\text { conversion - pre-XDR, } \\
\text { not on BDQ }\end{array}$ & $\begin{array}{l}\text { median time in weeks, assuming no death, } \\
\text { default, or acquired resistance }\end{array}$ & $\begin{array}{l}16.85 \text { (18 allowing } \\
\text { for acquired } \\
\text { resistance) }\end{array}$ & $\begin{array}{l}\text { [2] (HR 0.6 FQ-res, } 0.8 \\
\text { 2LI-res), [4] }\end{array}$ \\
\hline MedianCCXDR & $\begin{array}{l}\text { Median time to culture } \\
\text { conversion - XDR, not } \\
\text { on BDQ }\end{array}$ & $\begin{array}{l}\text { median time in weeks, assuming no death, } \\
\text { default, or acquired resistance }\end{array}$ & 26 & $\begin{array}{l}{[3](183 \text { days }),[2](\mathrm{HR}} \\
0.52)\end{array}$ \\
\hline $\begin{array}{l}\text { pAcquireBDQ_M } \\
\text { DR }\end{array}$ & $\begin{array}{l}\text { Weekly probability of } \\
\text { acquiring BDQ } \\
\text { resistance from MDR }\end{array}$ & $\begin{array}{l}\text { Probability of ever acquiring BDQ } \\
\text { resistance (initially MDR) is } \\
\text { RiskResBDQXDR/16 }\end{array}$ & $\begin{array}{l}\text { See calculation } \\
\text { page }\end{array}$ & $\begin{array}{l}\text { Assuming each new drug } \\
\text { reduces the risk of } \\
\text { resistance 4-fold, similar to } \\
\text { [5] }\end{array}$ \\
\hline $\begin{array}{l}\text { pAcquireBDQ_Pre } \\
\text { XDR }\end{array}$ & $\begin{array}{l}\text { Weekly probability of } \\
\text { acquiring BDQ } \\
\text { resistance from pre- } \\
\text { XDR }\end{array}$ & $\begin{array}{l}\text { Probability of acquiring BDQ resistance } \\
\text { (initially pre-XDR) is } \\
\text { RiskResBDQXDR/4 }\end{array}$ & $\begin{array}{l}\text { See calculation } \\
\text { page }\end{array}$ & {$[5]$} \\
\hline
\end{tabular}




\begin{tabular}{|c|c|c|c|c|}
\hline $\begin{array}{l}\text { pAcquireBDQ_XD } \\
\text { R }\end{array}$ & $\begin{array}{l}\text { Weekly probability of } \\
\text { acquiring BDQ } \\
\text { resistance from XDR }\end{array}$ & $\begin{array}{l}\text { Probability of ever acquiring BDQ } \\
\text { resistance (initially XDR) is } \\
\text { RiskResBDQXDR }\end{array}$ & $\begin{array}{l}\text { See calculation } \\
\text { page }\end{array}$ & {$[5]$} \\
\hline pAcquirePreXDR & $\begin{array}{l}\text { Weekly probability of } \\
\text { acquiring pre-XDR } \\
\text { (from MDR, not on } \\
\text { BDQ) }\end{array}$ & $\begin{array}{l}\text { Probability of ever acquiring FQ-R or } \\
\text { 2LI-R (initially MDR, not on BDQ) prior } \\
\text { to death, default, stopping treatment, or } \\
\text { conversion is } 0.065 \text { each }(10.3 \% \text { either })\end{array}$ & $\begin{array}{l}\text { See calculation } \\
\text { page }\end{array}$ & [5] \\
\hline $\begin{array}{l}\text { pAcquirePreXDR_- } \\
\text { BDQ }\end{array}$ & $\begin{array}{l}\text { Weekly probability of } \\
\text { acquiring pre-XDR } \\
\text { (from MDR, while on } \\
\text { BDQ) }\end{array}$ & $\begin{array}{l}\text { Probability of ever acquiring FQ-R or } \\
\text { 2LI-R (initially MDR, on BDQ) prior to } \\
\text { death, default, stopping treatment, or } \\
\text { conversion is } 0.065 / \text { ResProtectionBDQ }\end{array}$ & $\begin{array}{l}\text { See calculation } \\
\text { page }\end{array}$ & {$[5]$} \\
\hline pAcquireXDR & $\begin{array}{l}\text { Weekly probability of } \\
\text { acquiring XDR (from } \\
\text { pre-XDR, not on } \\
\text { BDQ) }\end{array}$ & $\begin{array}{l}\text { Probability of ever acquiring XDR } \\
\text { (initially pre-XDR, not on BDQ) prior to } \\
\text { death, default, stopping treatment, or } \\
\text { conversion is } 0.26\end{array}$ & $\begin{array}{l}\text { See calculation } \\
\text { page }\end{array}$ & [5] \\
\hline $\begin{array}{l}\text { pAcquireXDR_BD } \\
Q\end{array}$ & $\begin{array}{l}\text { Weekly probability of } \\
\text { acquiring XDR (from } \\
\text { pre-XDR, while on } \\
\text { BDQ) }\end{array}$ & $\begin{array}{l}\text { Probability of ever acquiring XDR } \\
\text { (initially pre-XDR, on BDQ) prior to } \\
\text { death, default, stopping treatment, or } \\
\text { conversion is } 0.26 / \text { ResProtectionBDQ }\end{array}$ & $\begin{array}{l}\text { See calculation } \\
\text { page }\end{array}$ & {$[5]$} \\
\hline pConvertMDR & $\begin{array}{l}\text { Weekly probability of } \\
\text { culture conversion - } \\
\text { MDR on BR }\end{array}$ & $\begin{array}{l}\text { Assuming median time to culture } \\
\text { conversion in the absence of death, } \\
\text { default, or acquired resistance would be } \\
\text { MedianCCMDR }\end{array}$ & $\begin{array}{l}\text { See calculation } \\
\text { page }\end{array}$ & NA \\
\hline $\begin{array}{l}\text { pConvertMDR_BD } \\
Q\end{array}$ & $\begin{array}{l}\text { Weekly probability of } \\
\text { culture conversion - } \\
\text { MDR on BDQ }\end{array}$ & $\begin{array}{l}\text { Assuming median time to culture } \\
\text { conversion in the absence of death, } \\
\text { default, or acquired resistance would be } \\
\text { MedianCCMDR*BDQMedianCCRR }\end{array}$ & $\begin{array}{l}\text { See calculation } \\
\text { page }\end{array}$ & NA \\
\hline pConvertPreXDR & $\begin{array}{l}\text { Weekly probability of } \\
\text { culture conversion - } \\
\text { PreXDR on BR }\end{array}$ & $\begin{array}{l}\text { Assuming median time to culture } \\
\text { conversion in the absence of death, } \\
\text { default, or acquired resistance would be } \\
\text { MedianCCPreXDR }\end{array}$ & $\begin{array}{l}\text { See calculation } \\
\text { page }\end{array}$ & NA \\
\hline $\begin{array}{l}\text { pConvertPreXDR_ } \\
\text { BDQ }\end{array}$ & $\begin{array}{l}\text { Weekly probability of } \\
\text { culture conversion - } \\
\text { PreXDR on BDQ }\end{array}$ & $\begin{array}{l}\text { Assuming median time to culture } \\
\text { conversion in the absence of death, } \\
\text { default, or acquired resistance would be } \\
\text { MedianCCPreXDR*BDQMedianCCRR }\end{array}$ & $\begin{array}{l}\text { See calculation } \\
\text { page }\end{array}$ & NA \\
\hline pConvertXDR & $\begin{array}{l}\text { Weekly probability of } \\
\text { culture conversion - } \\
\text { XDR on BR }\end{array}$ & $\begin{array}{l}\text { Assuming median time to culture } \\
\text { conversion in the absence of death, } \\
\text { default, or acquired resistance would be } \\
\text { MedianCCXDR }\end{array}$ & $\begin{array}{l}\text { See calculation } \\
\text { page }\end{array}$ & NA \\
\hline
\end{tabular}




\begin{tabular}{|c|c|c|c|c|}
\hline $\begin{array}{l}\text { pConvertXDR_BD } \\
\mathrm{Q}\end{array}$ & $\begin{array}{l}\text { Weekly probability of } \\
\text { culture conversion - } \\
\text { XDR on BDQ }\end{array}$ & $\begin{array}{l}\text { Assuming median time to culture } \\
\text { conversion in the absence of death, } \\
\text { default, or acquired resistance would be } \\
\text { MedianCCXDR*BDQMedianCCRR }\end{array}$ & $\begin{array}{l}\text { See calculation } \\
\text { page }\end{array}$ & NA \\
\hline pCure & $\begin{array}{l}\text { Weekly probability of } \\
\text { moving from "culture } \\
\text { negative" (high relapse } \\
\text { risk post-treatment) to } \\
\text { "cure" }\end{array}$ & $\begin{array}{l}\text { Reflects relapse probabilities that depend } \\
\text { on regimen and resistance pattern }\end{array}$ & $\begin{array}{l}\text { See calculation } \\
\text { page }\end{array}$ & NA \\
\hline pCureUntreated & $\begin{array}{l}\text { Weekly probability of } \\
\text { moving from "culture } \\
\text { positive" to "cure" for } \\
\text { untreated individuals }\end{array}$ & $\begin{array}{l}\text { Assumes a case fatality rate of } 60 \% \text { for } \\
\text { untreated TB }\end{array}$ & $.005 /(1-\mathrm{pDie})$ & {$[6]$} \\
\hline pDieBDQ & $\begin{array}{l}\text { Weekly probability of } \\
\text { dying from adverse } \\
\text { effects related to BDQ } \\
\text { administration }\end{array}$ & $\begin{array}{l}\text { Assume constant throughout treatment for } \\
\text { all patients who have ever received BDQ, } \\
\text { relfecting long half-life }\end{array}$ & $\begin{array}{l}\text { See calculation } \\
\text { page }\end{array}$ & NA \\
\hline pDieTreatedTB & $\begin{array}{l}\text { Weekly excess } \\
\text { probability of dying } \\
\text { from TB while culture } \\
\text { positive on treatment }\end{array}$ & $\begin{array}{l}\text { Chosen such that } 16 \% \text { of people with } \\
\text { XDR on BR die prior to default or } \\
\text { treatment completion. Excess mortality } \\
\text { only applies while culture positive }\end{array}$ & $.0075+\mathrm{pDieWell}$ & $\begin{array}{l}\text { [7] (15\% of patients with } \\
\text { XDR TB die, sums to } \\
93 \%)\end{array}$ \\
\hline pDieUntreatedTB & $\begin{array}{l}\text { Weekly excess } \\
\text { probability of dying } \\
\text { from TB while culture } \\
\text { positive not on } \\
\text { treatment }\end{array}$ & $\begin{array}{l}\text { Assumed to be equal to pDieTreatedTB } \\
\text { (case fatality of untreated TB still }>> \\
\text { treated TB because excess mortality only } \\
\text { applies while culture positive) }\end{array}$ & $.0075+\mathrm{pDieWell}$ & [7] \\
\hline pDieWell & $\begin{array}{l}\text { Weekly probability of } \\
\text { dying - background } \\
\text { mortality }\end{array}$ & Varies according to age & $\begin{array}{l}\text { Taken from WHO } \\
\text { European region } \\
\text { male lifetables } \\
\text { from } 2013\end{array}$ & [8] \\
\hline pEnd & $\begin{array}{l}\text { Probability of stopping } \\
\text { treatment routinely }\end{array}$ & $\begin{array}{l}\text { Assume average treatment duration of } 21 \\
\text { months regardless of resistance pattern }\end{array}$ & $.011 /(1-\mathrm{pDie})$ & [9] \\
\hline pRelapse & $\begin{array}{l}\text { Weekly probability of } \\
\text { relapse after treatment } \\
\text { (for individuals } \\
\text { stopping treatment not } \\
\text { fully cured) }\end{array}$ & $\begin{array}{l}\text { Assume } 75 \% \text { of relapses occur within one } \\
\text { year }\end{array}$ & 0.0263 & {$[10-13]$} \\
\hline
\end{tabular}




\begin{tabular}{|c|c|c|c|c|}
\hline $\begin{array}{l}\text { pStartBDQPreXD } \\
\mathrm{R}\end{array}$ & $\begin{array}{l}\text { Weekly probability of } \\
\text { starting bedaquiline } \\
\text { for patients with } \\
\text { PreXDR TB receiving } \\
\text { BR }\end{array}$ & $\begin{array}{l}0 \text { if ineligible for BDQ; otherwise assume } \\
\text { takes an average of } 13 \text { weeks for DST } \\
\text { results } \\
\text { RDT scenario: } 0 \text { if ineligible for BDQ, } 1 \\
\text { if eligible }\end{array}$ & $\begin{array}{l}0.077 /((1- \\
\text { pDie }) *(1- \\
\text { pDefault }) *(1- \\
\text { pEnd }))\end{array}$ & [9] \\
\hline pStartBDQXDR & $\begin{array}{l}\text { Weekly probability of } \\
\text { starting bedaquiline } \\
\text { for patients with XDR } \\
\text { TB receiving BR } \\
\end{array}$ & $\begin{array}{l}0 \text { if ineligible for BDQ; otherwise assume } \\
\text { takes an average of } 13 \text { weeks for DST } \\
\text { results } \\
\text { RDT scenario: } 0 \text { if ineligible, } 1 \text { if eligible }\end{array}$ & $\begin{array}{l}0.077 /((1- \\
\text { pDie)*(1- } \\
\text { pDefault)*(1- } \\
\text { pEnd }))\end{array}$ & [9] \\
\hline pStartTreat & $\begin{array}{l}\text { Weekly probability of } \\
\text { starting treatment for } \\
\text { untreated, culture } \\
\text { positive individuals }\end{array}$ & $\begin{array}{l}\text { Assume average } 1.5 \text { years ( } 78 \text { weeks) } \\
\text { prior to starting treatment }\end{array}$ & $\begin{array}{l}0.0128 /((1- \\
\text { pDie)*(1- } \\
\text { pCureUntreated }))\end{array}$ & $\begin{array}{l}\text { [14] (1.15 years smear+, } \\
2.39 \text { years all confirmed } \\
\text { TB among HIV- } \\
\text { individuals. We have } \\
\text { chosen an intermediate } \\
\text { value) }\end{array}$ \\
\hline pStartTreatBDQ & $\begin{array}{l}\text { Probability of } \\
\text { receiving BDQ from } \\
\text { the initiation of MDR } \\
\text { TB treatment } \\
\end{array}$ & $\begin{array}{l}1 \text { if all people with MDR are eligible for } \\
\text { BDQ; } 0 \text { otherwise } \\
\text { RDT scenario: } 0 \text { if specific resistance } \\
\text { pattern ineligible for BDQ, } 1 \text { if eligible }\end{array}$ & GetBDQMDR & NA \\
\hline pStartTreatBR & $\begin{array}{l}\text { Probability of } \\
\text { receiving BR only } \\
\text { from intiation of MDR } \\
\text { TB treatment }\end{array}$ & $\begin{array}{l}0 \text { if all people with MDR are eligible for } \\
\text { BDQ; } 1 \text { otherwise } \\
\text { RDT scenario: } 0 \text { if specific resistance } \\
\text { pattern eligible for BDQ, } 1 \text { if ineligible }\end{array}$ & 1-GetBDQMDR & NA \\
\hline RelapsePropMDR & $\begin{array}{l}\text { Proportion of people } \\
\text { with MDR TB only } \\
\text { who ever relapse }\end{array}$ & NA & 0.04 & [15-17] \\
\hline $\begin{array}{l}\text { RelapsePropPreXD } \\
\text { R }\end{array}$ & $\begin{array}{l}\text { Proportion of people } \\
\text { with PreXDR TB only } \\
\text { who ever relapse }\end{array}$ & NA & 0.08 & [15] \\
\hline RelapsePropXDR & $\begin{array}{l}\text { Proportion of people } \\
\text { with XDR TB only } \\
\text { who ever relapse }\end{array}$ & NA & 0.16 & {$[15,18]$} \\
\hline RiskPreXDRBDQ & $\begin{array}{l}\text { Risk of ever } \\
\text { developing PreXDR } \\
\text { for individuals with } \\
\text { MDR TB receiving } \\
\text { BDQ }\end{array}$ & $\begin{array}{l}\text { Assuming BDQ reduces the risk of } \\
\text { resistance to any fluoroquinolone or any } \\
\text { second-line injectable by } \\
\text { ResProtectionBDQ }\end{array}$ & $\begin{array}{l}\text { See calculation } \\
\text { page }\end{array}$ & NA \\
\hline
\end{tabular}




\begin{tabular}{|c|c|c|c|c|}
\hline SecMultiUntreated & $\begin{array}{l}\text { Ratio of infectiousness } \\
\text { of untreated to treated } \\
\text { individuals with } \\
\text { culture positive TB } \\
\end{array}$ & $\begin{array}{l}\text { Assuming similar to the infectiousness of } \\
\text { smear-positive vs. smear-negative } \\
\text { individuals }\end{array}$ & 5 & [19-21] \\
\hline uLE & $\begin{array}{l}\text { Weekly utility when } \\
\text { calculating life } \\
\text { expectancy }\end{array}$ & Fraction of a year & $1 / 52$ & NA \\
\hline uSec_MDR & $\begin{array}{l}\text { Weekly utility when } \\
\text { calculating secondary } \\
\text { MDR cases (on } \\
\text { treatment, culture } \\
\text { positive) }\end{array}$ & $\begin{array}{l}10 \text { persons infected per year if smear } \\
\text { positive* } 0.1 \text { chance of progressing to } \\
\text { active TB*.7 fitness } \\
\text { MDR*( } 1 / \text { SecMultiUntreated })\end{array}$ & $0.14 / 52$ & [21-27] \\
\hline uSec_MDR_BDQ & $\begin{array}{l}\text { Weekly utility when } \\
\text { calculating secondary } \\
\text { MDR+BDQR cases } \\
\text { (on treatment, culture } \\
\text { positive) }\end{array}$ & $\begin{array}{l}10 \text { persons infected per year if smear } \\
\text { positive* } 0.1 \text { chance of progressing to } \\
\text { active TB*. } 7 \text { fitness } \\
\text { MDR*FitCostBDQR } \\
(1 / \text { SecMultiUntreated })\end{array}$ & $\begin{array}{l}0.14 * \text { FitCostBDQ } \\
\mathrm{R} / 52\end{array}$ & {$[21-27]$} \\
\hline uSec_PreX_BDQ & $\begin{array}{l}\text { Weekly utility when } \\
\text { calculating secondary } \\
\text { PreXDR+BDQR cases } \\
\text { (on treatment, culture } \\
\text { positive) }\end{array}$ & $\begin{array}{l}10 \text { persons infected per year if smear } \\
\text { positive* } 0.1 \text { chance of progressing to } \\
\text { active } \mathrm{TB}^{*} .63 \text { fitness PreXDRDR* } \\
\text { FitCostBDQR* (1/SecMultiUntreated) }\end{array}$ & $\begin{array}{l}.126^{*} \text { FitCostBDQ } \\
\mathrm{R} / 52\end{array}$ & {$[21-27]$} \\
\hline uSec_PreXDR & $\begin{array}{l}\text { Weekly utility when } \\
\text { calculating secondary } \\
\text { PreXDR cases (on } \\
\text { treatment, culture } \\
\text { positive) }\end{array}$ & $\begin{array}{l}10 \text { persons infected per year if smear } \\
\text { positive* } 0.1 \text { chance of progressing to } \\
\text { active TB* } 63 \text { fitness PreXDR* } \\
\text { (1/SecMultiUntreated) }\end{array}$ & $.126 / 52$ & {$[21-27]$} \\
\hline uSec_XDR & $\begin{array}{l}\text { Weekly utility when } \\
\text { calculating secondary } \\
\text { XDR cases (on } \\
\text { treatment, culture } \\
\text { positive) }\end{array}$ & $\begin{array}{l}10 \text { persons infected per year if smear } \\
\text { positive* } 0.1 \text { chance of progressing to } \\
\text { active } \mathrm{TB}^{*} .51 \text { fitness } \\
\text { XDR* }(1 / \text { SecMultiUntreated })\end{array}$ & $0.113 / 52$ & {$[21-27]$} \\
\hline uSec_XDR_BDQR & $\begin{array}{l}\text { Weekly utility when } \\
\text { calculating secondary } \\
\text { XDR+BDQR cases } \\
\text { (on treatment, culture } \\
\text { positive) }\end{array}$ & $\begin{array}{l}10 \text { persons infected per year if smear } \\
\text { positive* } 0.1 \text { chance of progressing to } \\
\text { active TB*. } 51 \text { fitness } \\
\text { XDR*FitCostBDQR* } \\
(1 / \text { SecMultiUntreated })\end{array}$ & $\begin{array}{l}0.113 * \text { FitCostBD } \\
\mathrm{QR} / 52\end{array}$ & {$[21,22,27-30]$} \\
\hline
\end{tabular}




\begin{tabular}{|l|l|l|l|l|}
\hline GetBDQMDR & $\begin{array}{l}\text { Whether people with } \\
\text { MDR TB are eligible } \\
\text { for BDQ }\end{array}$ & Varies depending on treatment scenario & 1 if yes, 0 if no & NA \\
\hline GetBDQPreXDR & $\begin{array}{l}\text { Whether people with } \\
\text { PreXDR TB are } \\
\text { eligible for BDQ }\end{array}$ & Varies depending on treatment scenario & 1 if yes, 0 if no & NA \\
\hline GetBDQXDR & $\begin{array}{l}\text { Whether people with } \\
\text { XDR TB are eligible } \\
\text { for BDQ }\end{array}$ & Varies depending on treatment scenario & 1 if yes, 0 if no & NA \\
\hline pDefault & $\begin{array}{l}\text { Weekly default } \\
\text { probability }\end{array}$ & $\begin{array}{l}\text { Constant throughout treatment, value } \\
\text { depends on regimen. Baseline is chosen } \\
\text { such that } 17.3 \% \text { of people with XDR on } \\
\text { BR default prior to death or stopping } \\
\text { treatment. }\end{array}$ & See TreeAge file & {$[7]$} \\
\hline pDie & $\begin{array}{l}\text { Weekly probability of } \\
\text { death }\end{array}$ & $\begin{array}{l}\text { Depends on whether the individual is } \\
\text { culture positive or receiving bedaquiline } \\
\text { and their age }\end{array}$ & $\begin{array}{l}\text { Sum of relevant } \\
\text { probabilities }\end{array}$ & NA \\
\hline RelapseProp & $\begin{array}{l}\text { Proportion of people } \\
\text { who would relapse } \\
\text { post-treatment }\end{array}$ & $\begin{array}{l}\text { Depends on the individual's regimen and } \\
\text { resistance pattern }\end{array}$ & See TreeAge file & NA \\
\hline
\end{tabular}




\section{COMPLETE LIST OF MODEL TRANSITIONS}

From States Receiving Bedaquiline

\section{MDR, Bedaquiline-Sensitive, On Bedaquiline, Culture+}

Die -> Death

Stop treatment (routine) -> MDR, Bedaquiline-Sensitive, Not on Treatment, Culture+

Default -> MDR, Bedaquiline-Sensitive, Not on Treatment, Culture+

Convert -> MDR, Bedaquiline-Sensitive, On Bedaquiline, Culture-

Acquire PreXDR -> PreXDR, Bedaquiline-Sensitive, On Bedaquiline, Culture+

Acquire BDQR -> MDR, Bedaquiline-Resistant, On Bedaquiline, Culture+

Remain in same state

\section{MDR, Bedaquiline-Resistant, On Bedaquiline, Culture+}

Die -> Death

Stop treatment (routine) -> MDR, Bedaquiline-Resistant, Not on Treatment, Culture+

Default -> MDR, Bedaquiline-Resistant, Not on Treatment, Culture+

Convert -> MDR, Bedaquiline-Resistant, On Bedaquiline, Culture-

Acquire PreXDR -> PreXDR, Bedaquiline-Resistant, On Bedaquiline, Culture+

Remain in same state

\section{MDR, Bedaquiline-Sensitive, On Bedaquiline, Culture-}

Die -> Death

Stop treatment (routine) -> MDR, Bedaquiline-Sensitive, Not on Treatment, Culture-

Default -> MDR, Bedaquiline-Sensitive, Not on Treatment, Culture-

Cure -> Any Resistance, On Bedaquiline, Cured

Remain in same state

\section{MDR, Bedaquiline-Resistant, On Bedaquiline, Culture-}

Die -> Death

Stop treatment (routine) -> MDR, Bedaquiline-Resistant, Not on Treatment, Culture-

Default -> MDR, Bedaquiline-Resistant, Not on Treatment, Culture-

Cure -> Any Resistance, On Bedaquiline, Cured 
Remain in same state

PreXDR, Bedaquiline-Sensitive, On Bedaquiline, Culture+

Die $->$ Death

Stop treatment (routine) -> PreXDR, Bedaquiline-Sensitive, Not on Treatment, Culture+

Default -> PreXDR, Bedaquiline-Sensitive, Not on Treatment, Culture+

Convert -> PreXDR, Bedaquiline-Sensitive, On Bedaquiline, Culture-

Acquire XDR -> XDR, Bedaquiline-Sensitive, On Bedaquiline, Culture+

Acquire BDQR -> PreXDR, Bedaquiline-Resistant, On Bedaquiline, Culture+

Remain in same state

PreXDR, Bedaquiline-Resistant, On Bedaquiline, Culture+

Die -> Death

Stop treatment (routine) -> PreXDR, Bedaquiline-Resistant, Not on Treatment, Culture+

Default -> PreXDR, Bedaquiline-Resistant, Not on Treatment, Culture+

Convert -> PreXDR, Bedaquiline-Resistant, On Bedaquiline, Culture-

Acquire XDR -> XDR, Bedaquiline-Resistant, On Bedaquiline, Culture+

Remain in same state

PreXDR, Bedaquiline-Sensitive, On Bedaquiline, Culture-

Die -> Death

Stop treatment (routine) -> PreXDR, Bedaquiline-Sensitive, Not on Treatment, Culture-

Default -> PreXDR, Bedaquiline-Sensitive, Not on Treatment, Culture-

Cure -> Any Resistance, On Bedaquiline, Cured

Remain in same state

PreXDR, Bedaquiline-Resistant, On Bedaquiline, Culture-

Die -> Death

Stop treatment (routine) -> PreXDR, Bedaquiline-Resistant, Not on Treatment, Culture-

Default -> PreXDR, Bedaquiline-Resistant, Not on Treatment, Culture-

Cure -> Any Resistance, On Bedaquiline, Cured

Remain in same state 
XDR, Bedaquiline-Sensitive, On Bedaquiline, Culture+

Die -> Death

Stop treatment (routine) -> XDR, Bedaquiline-Sensitive, Not on Treatment, Culture+

Default -> XDR, Bedaquiline-Sensitive, Not on Treatment, Culture+

Convert -> XDR, Bedaquiline-Sensitive, On Bedaquiline, Culture-

Acquire BDQR -> XDR, Bedaquiline-Resistant, On Bedaquiline, Culture+

Remain in same state

XDR, Bedaquiline-Resistant, On Bedaquiline, Culture+

Die -> Death

Stop treatment (routine) -> XDR, Bedaquiline-Resistant, Not on Treatment, Culture+

Default -> XDR, Bedaquiline-Resistant, Not on Treatment, Culture+

Convert -> XDR, Bedaquiline-Resistant, On Bedaquiline, Culture-

Remain in same state

XDR, Bedaquiline-Sensitive, On Bedaquiline, Culture-

Die -> Death

Stop treatment (routine) -> XDR, Bedaquiline-Sensitive, Not on Treatment, Culture-

Default -> XDR, Bedaquiline-Sensitive, Not on Treatment, Culture-

Cure -> Any Resistance, On Bedaquiline, Cured

Remain in same state

XDR, Bedaquiline-Resistant, On Bedaquiline, Culture-

Die -> Death

Stop treatment (routine) -> XDR, Bedaquiline-Resistant, Not on Treatment, Culture-

Default -> XDR, Bedaquiline-Resistant, Not on Treatment, Culture-

Cure -> Any Resistance, On Bedaquiline, Cured

Remain in same state

Any Resistance, On Bedaquiline, Cured

Die -> Death

Stop treatment (routine) -> Any Resistance, Not on Treatment, Cured 
Default -> Any Resistance, Not on Treatment, Cured

Remain in same state

\section{From States Receiving OBR}

\section{MDR, Bedaquiline-Sensitive, On OBR, Culture+}

Die -> Death

Stop treatment (routine) -> MDR, Bedaquiline-Sensitive, Not on Treatment, Culture+

Default -> MDR, Bedaquiline-Sensitive, Not on Treatment, Culture+

Convert -> MDR, Bedaquiline-Sensitive, On OBR, Culture-

Acquire PreXDR -> PreXDR, Bedaquiline-Sensitive, On OBR, Culture+

Remain in same state

\section{MDR, Bedaquiline-Resistant, On OBR, Culture+}

Die -> Death

Stop treatment (routine) -> MDR, Bedaquiline-Resistant, Not on Treatment, Culture+

Default -> MDR, Bedaquiline-Resistant, Not on Treatment, Culture+

Convert -> MDR, Bedaquiline-Resistant, On OBR, Culture-

Acquire PreXDR -> PreXDR, Bedaquiline-Resistant, On OBR, Culture+

Remain in same state

\section{MDR, Bedaquiline-Sensitive, On OBR, Culture-}

Die -> Death

Stop treatment (routine) -> MDR, Bedaquiline-Sensitive, Not on Treatment, Culture-

Default -> MDR, Bedaquiline-Sensitive, Not on Treatment, Culture-

Cure -> MDR, On OBR, Cured

Remain in same state

\section{MDR, Bedaquiline-Resistant, On OBR, Culture-}

Die -> Death

Stop treatment (routine) -> MDR, Bedaquiline-Resistant, Not on Treatment, Culture-

Default -> MDR, Bedaquiline-Resistant, Not on Treatment, Culture-

Cure -> MDR, On OBR, Cured 
Remain in same state

PreXDR, Bedaquiline-Sensitive, On OBR, Culture+

Die -> Death

Stop treatment (routine) -> PreXDR, Bedaquiline-Sensitive, Not on Treatment, Culture+

Default -> PreXDR, Bedaquiline-Sensitive, Not on Treatment, Culture+

Convert \& Start BDQ -> PreXDR, Bedaquiline-Sensitive, On Bedaquiline, Culture-

Convert \& Continue OBR -> PreXDR, Bedaquiline-Sensitive, On OBR, Culture-

Acquire XDR \& Start BDQ -> XDR, Bedaquiline-Sensitive, On Bedaquiline, Culture+

Acquire XDR \& Continue OBR -> XDR, Bedaquiline-Sensitive, On OBR, Culture+

Start BDQ -> PreXDR, Bedaquiline-Sensitive, On Bedaquiline, Culture+

Remain in same state

PreXDR, Bedaquiline-Resistant, On OBR, Culture+

Die -> Death

Stop treatment (routine) -> PreXDR, Bedaquiline-Resistant, Not on Treatment, Culture+

Default -> PreXDR, Bedaquiline-Resistant, Not on Treatment, Culture+

Convert \& Start BDQ -> PreXDR, Bedaquiline-Resistant, On Bedaquiline, Culture-

Convert \& Continue OBR -> PreXDR, Bedaquiline-Resistant, On OBR, Culture-

Acquire XDR \& Start BDQ -> XDR, Bedaquiline-Resistant, On Bedaquiline, Culture+

Acquire XDR \& Continue OBR -> XDR, Bedaquiline-Resistant, On OBR, Culture+

Start BDQ -> PreXDR, Bedaquiline-Resistant, On Bedaquiline, Culture+

Remain in same state

PreXDR, Bedaquiline-Sensitive, On OBR, Culture-

Die -> Death

Stop treatment (routine) -> PreXDR, Bedaquiline-Sensitive, Not on Treatment, Culture-

Default -> PreXDR, Bedaquiline-Sensitive, Not on Treatment, Culture-

Cure \& Start BDQ -> Any Resistance, On Bedaquiline, Cured

Cure \& Continue OBR -> PreXDR, On OBR, Cured

Start BDQ -> PreXDR, Bedaquiline-Sensitive, On Bedaquiline, Culture- 
Remain in same state

PreXDR, Bedaquiline-Resistant, On OBR, Culture-

Die -> Death

Stop treatment (routine) -> PreXDR, Bedaquiline-Resistant, Not on Treatment, Culture-

Default -> PreXDR, Bedaquiline-Resistant, Not on Treatment, Culture-

Cure \& Start BDQ -> Any Resistance, On Bedaquiline, Cured

Cure \& Continue OBR -> PreXDR, On OBR, Cured

Start BDQ -> PreXDR, Bedaquiline-Resistant, On Bedaquiline, Culture-

Remain in same state

XDR, Bedaquiline-Sensitive, On OBR, Culture+

Die -> Death

Stop treatment (routine) -> XDR, Bedaquiline-Sensitive, Not on Treatment, Culture+

Default -> XDR, Bedaquiline-Sensitive, Not on Treatment, Culture+

Convert \& Start BDQ -> XDR, Bedaquiline-Sensitive, On Bedaquiline, Culture-

Convert \& Continue OBR -> XDR, Bedaquiline-Sensitive, On OBR, Culture-

Start BDQ -> XDR, Bedaquiline-Sensitive, On Bedaquiline, Culture+

Remain in same state

XDR, Bedaquiline-Resistant, On OBR, Culture+

Die -> Death

Stop treatment (routine) -> XDR, Bedaquiline-Resistant, Not on Treatment, Culture+

Default -> XDR, Bedaquiline-Resistant, Not on Treatment, Culture+

Convert \& Start BDQ -> XDR, Bedaquiline-Resistant, On Bedaquiline, Culture-

Convert \& Continue OBR -> XDR, Bedaquiline-Resistant, On OBR, Culture-

Start BDQ -> XDR, Bedaquiline-Resistant, On Bedaquiline, Culture+

Remain in same state

XDR, Bedaquiline-Sensitive, On OBR, Culture-

Die -> Death

Stop treatment (routine) -> XDR, Bedaquiline-Sensitive, Not on Treatment, Culture- 
Default -> XDR, Bedaquiline-Sensitive, Not on Treatment, Culture-

Cure \& Start BDQ -> Any Resistance, On Bedaquiline, Cured

Cure \& Continue OBR -> XDR, On OBR, Cured

Start BDQ -> XDR, Bedaquiline-Sensitive, On Bedaquiline, Culture-

Remain in same state

XDR, Bedaquiline-Resistant, On OBR, Culture-

Die -> Death

Stop treatment (routine) -> XDR, Bedaquiline-Resistant, Not on Treatment, Culture-

Default -> XDR, Bedaquiline-Resistant, Not on Treatment, Culture-

Cure \& Start BDQ -> Any Resistance, On Bedaquiline, Cured

Cure \& Continue OBR -> XDR, On OBR, Cured

Start BDQ -> XDR, Bedaquiline-Resistant, On Bedaquiline, Culture-

Remain in same state

\section{MDR, On OBR, Cured}

Die -> Death

Stop treatment (routine) -> Any Resistance, Not on Treatment, Cured

Default -> Any Resistance, Not on Treatment, Cured

Remain in same state

\section{PreXDR, On OBR, Cured}

Die -> Death

Stop treatment (routine) -> Any Resistance, Not on Treatment, Cured

Default -> Any Resistance, Not on Treatment, Cured

Start BDQ -> Any Resistance, On Bedaquiline, Cured

Remain in same state

\section{XDR, On OBR, Cured}

Die -> Death

Stop treatment (routine) -> Any Resistance, Not on Treatment, Cured

Default -> Any Resistance, Not on Treatment, Cured 
Start BDQ -> Any Resistance, On Bedaquiline, Cured

Remain in same state

From States Not on Treatment

MDR, Bedaquiline-Sensitive, Not on Treatment, Culture+

Die -> Death

Cure -> Any Resistance, Not on Treatment, Cured

Start treatment with OBR -> MDR, Bedaquiline-Sensitive, On OBR, Culture+

Start treatment with BDQ -> MDR, Bedaquiline-Sensitive, On Bedaquiline, Culture+

Remain in same state

MDR, Bedaquiline-Resistant, Not on Treatment, Culture+

Die -> Death

Cure -> Any Resistance, Not on Treatment, Cured

Start treatment with OBR -> MDR, Bedaquiline-Resistant, On OBR, Culture+

Start treatment with BDQ -> MDR, Bedaquiline-Resistant, On Bedaquiline, Culture+

Remain in same state

MDR, Bedaquiline-Sensitive, Not on Treatment, Culture-

Die -> Death

Relapse -> MDR, Bedaquiline-Sensitive, Not on Treatment, Culture+

Remain in same state

MDR, Bedaquiline-Resistant, Not on Treatment, Culture-

Die -> Death

Relapse -> MDR, Bedaquiline-Resistant, Not on Treatment, Culture+

Remain in same state

PreXDR, Bedaquiline-Sensitive, Not on Treatment, Culture +

Die -> Death

Cure -> Any Resistance, Not on Treatment, Cured

Start treatment with OBR -> PreXDR, Bedaquiline-Sensitive, On OBR, Culture+

Start treatment with BDQ -> PreXDR, Bedaquiline-Sensitive, On Bedaquiline, Culture+ 
Remain in same state

PreXDR, Bedaquiline-Resistant, Not on Treatment, Culture+

Die -> Death

Cure -> Any Resistance, Not on Treatment, Cured

Start treatment with OBR -> PreXDR, Bedaquiline-Resistant, On OBR, Culture+

Start treatment with BDQ -> PreXDR, Bedaquiline-Resistant, On Bedaquiline, Culture+

Remain in same state

PreXDR, Bedaquiline-Sensitive, Not on Treatment, Culture-

Die -> Death

Relapse -> PreXDR, Bedaquiline-Sensitive, Not on Treatment, Culture+

Remain in same state

PreXDR, Bedaquiline-Resistant, Not on Treatment, Culture-

Die -> Death

Relapse -> PreXDR, Bedaquiline-Resistant, Not on Treatment, Culture+

Remain in same state

XDR, Bedaquiline-Sensitive, Not on Treatment, Culture+

Die -> Death

Cure -> Any Resistance, Not on Treatment, Cured

Start treatment with OBR -> XDR, Bedaquiline-Sensitive, On OBR, Culture+

Start treatment with BDQ -> XDR, Bedaquiline-Sensitive, On Bedaquiline, Culture+

Remain in same state

XDR, Bedaquiline-Resistant, Not on Treatment, Culture+

Die -> Death

Cure -> Any Resistance, Not on Treatment, Cured

Start treatment with OBR -> XDR, Bedaquiline-Resistant, On OBR, Culture+

Start treatment with BDQ -> XDR, Bedaquiline-Resistant, On Bedaquiline, Culture+

Remain in same state

XDR, Bedaquiline-Sensitive, Not on Treatment, Culture- 
Die -> Death

Relapse -> XDR, Bedaquiline-Sensitive, Not on Treatment, Culture+

Remain in same state

XDR, Bedaquiline-Resistant, Not on Treatment, Culture-

Die -> Death

Relapse -> XDR, Bedaquiline-Resistant, Not on Treatment, Culture+

Remain in same state

Any Resistance, Not on Treatment, Cured

Die -> Death

Remain in same state

Death

Remain in same state

\section{REFERENCES}

1. Dalton T, Cegielski P, Akksilp S, Asencios L, Campos Caoili J, Cho SN et al. Prevalence of and risk factors for resistance to second-line drugs in people with multidrug-resistant tuberculosis in eight countries: a prospective cohort study. Lancet. 2012;380(9851):1406-17. doi:10.1016/s0140-6736(12)60734-x.

2. Kurbatova E, Gammino VM, Bayona J, Becerra MC, Danilovitz M, Falzon D et al. Predictors of sputum culture conversion among patients treated for multidrug-resistant tuberculosis. International Journal of Tuberculosis and Lung Disease. 2012;16:1335-43. doi:10.5588/ijtld.11.0811.

3. Shah NS, Pratt R, Armstrong L, Robison V, Castro KG, Cegielski JP. Extensively drug-resistant tuberculosis in the United States, 1993-2007. Jama. 2008;300(18):2153-60. doi:10.1001/jama.300.18.2153. 4. Diacon AH, Lounis N, Dannemann B. Multidrug-resistant tuberculosis and bedaquiline. The New England journal of medicine. 2014;371(25):2436. doi:10.1056/NEJMc1412235.

5. Cegielski JP, Dalton T, Yagui M, Wattanaamornkiet W, Volchenkov GV, Via LE et al. Extensive drug resistance acquired during treatment of multidrug-resistant tuberculosis. Clinical infectious diseases : an official publication of the Infectious Diseases Society of America. 2014;59(8):1049-63.

doi:10.1093/cid/ciu572.

6. Tiemersma EW, van der Werf MJ, Borgdorff MW, Williams BG, Nagelkerke NJD. Natural history of tuberculosis: duration and fatality of untreated pulmonary tuberculosis in HIV negative patients: a systematic review. PloS one. 2011;6:e17601. doi:10.1371/journal.pone.0017601.

7. Falzon D, Gandhi N, Migliori GB, Sotgiu G, Cox H, Holtz TH et al. Resistance to fluoroquinolones and second-line injectable drugs: impact on MDR-TB outcomes. The European respiratory journal : official journal of the European Society for Clinical Respiratory Physiology. 2012:1-24.

doi:10.1183/09031936.00134712.

8. World Health Organization. Life tables by WHO region: Europe. In: Global Health Observatory data repository. 2015. Accessed June 112015.

9. World Health Organization. Guidelines for the programmatic management of drug-resistant tuberculosis: 2011 update. Geneva2011.

10. Glynn JR, Murray J, Bester A, Nelson G, Shearer S, Sonnenberg P. High rates of recurrence in HIVinfected and HIV-uninfected patients with tuberculosis. The Journal of infectious diseases.

2010;201(5):704-11. doi:10.1086/650529. 
11. Guerra-Assuncao JA, Houben RM, Crampin AC, Mzembe T, Mallard K, Coll F et al. Recurrence due to relapse or reinfection with Mycobacterium tuberculosis: a whole-genome sequencing approach in a large, population-based cohort with a high HIV infection prevalence and active follow-up. The Journal of infectious diseases. 2015;211(7):1154-63. doi:10.1093/infdis/jiu574.

12. Marx FM, Dunbar R, Enarson DA, Williams BG, Warren RM, van der Spuy GD et al. The temporal dynamics of relapse and reinfection tuberculosis after successful treatment: a retrospective cohort study. Clinical infectious diseases : an official publication of the Infectious Diseases Society of America. 2014;58(12):1676-83. doi:10.1093/cid/ciu186.

13. Nunn AJ, Phillips PP, Mitchison DA. Timing of relapse in short-course chemotherapy trials for tuberculosis. The international journal of tuberculosis and lung disease : the official journal of the International Union against Tuberculosis and Lung Disease. 2010;14(2):241-2.

14. Corbett EL, Charalambous S, Moloi VM, Fielding K, Grant AD, Dye C et al. Human immunodeficiency virus and the prevalence of undiagnosed tuberculosis in African gold miners. American journal of respiratory and critical care medicine. 2004;170(6):673-9. doi:10.1164/rccm.200405-590OC. 15. Chiang CY, Enarson DA, Yu MC, Bai KJ, Huang RM, Hsu CJ et al. Outcome of pulmonary multidrugresistant tuberculosis: a 6-yr follow-up study. The European respiratory journal. 2006;28(5):980-5. doi:10.1183/09031936.06.00125705.

16. Johnston JC, Khan FA, Dowdy DW. Reducing relapse in tuberculosis treatment: is it time to reassess WHO treatment guidelines? The international journal of tuberculosis and lung disease : the official journal of the International Union against Tuberculosis and Lung Disease. 2015;19(6):624.

doi:10.5588/ijtld.15.0224.

17. Gelmanova IY, Ahmad Khan F, Becerra MC, Zemlyanaya NA, Unakova IA, Andreev YG et al. Low rates of recurrence after successful treatment of multidrug-resistant tuberculosis in Tomsk, Russia. The international journal of tuberculosis and lung disease : the official journal of the International Union against Tuberculosis and Lung Disease. 2015;19(4):399-405. doi:10.5588/ijtld.14.0415.

18. Menzies D, Benedetti A, Paydar A, Martin I, Royce S, Pai M et al. Effect of duration and intermittency of rifampin on tuberculosis treatment outcomes: a systematic review and meta-analysis. PLoS medicine. 2009;6(9):e1000146. doi:10.1371/journal.pmed.1000146.

19. Tostmann A, Kik SV, Kalisvaart NA, Sebek MM, Verver S, Boeree MJ et al. Tuberculosis transmission by patients with smear-negative pulmonary tuberculosis in a large cohort in the Netherlands. Clinical infectious diseases : an official publication of the Infectious Diseases Society of America.

2008;47(9):1135-42. doi:10.1086/591974.

20. Behr MA, Warren SA, Salamon H, Hopewell PC, Ponce de Leon A, Daley CL et al. Transmission of Mycobacterium tuberculosis from patients smear-negative for acid-fast bacilli. Lancet. 1999;353(9151):444-9.

21. Styblo K. Epidemiology of Tuberculosis: Selected Papers. The Hague: Royal Netherlands Tuberculosis Foundation (KNCV); 1991.

22. Trunz BB, Fine P, Dye C. Effect of BCG vaccination on childhood tuberculous meningitis and miliary tuberculosis worldwide: a meta-analysis and assessment of cost-effectiveness. Lancet.

2006;367(9517):1173-80. doi:10.1016/s0140-6736(06)68507-3.

23. Gagneux S, Long CD, Small PM, Van T, Schoolnik GK, Bohannan BJ. The competitive cost of antibiotic resistance in Mycobacterium tuberculosis. Science (New York, NY). 2006;312(5782):1944-6. doi:10.1126/science.1124410.

24. Luciani F, Sisson SA, Jiang H, Francis AR, Tanaka MM. The epidemiological fitness cost of drug resistance in Mycobacterium tuberculosis. Proceedings of the National Academy of Sciences of the United States of America. 2009;106(34):14711-5. doi:10.1073/pnas.0902437106.

25. Bottger EC, Springer B. Tuberculosis: drug resistance, fitness, and strategies for global control. European journal of pediatrics. 2008;167(2):141-8. doi:10.1007/s00431-007-0606-9.

26. Grandjean L, Gilman RH, Martin L, Soto E, Castro B, Lopez S et al. Transmission of MultidrugResistant and Drug-Susceptible Tuberculosis within Households: A Prospective Cohort Study. PLoS medicine. 2015;12(6):e1001843; discussion e. doi:10.1371/journal.pmed.1001843.

27. Vynnycky E, Fine PE. The natural history of tuberculosis: the implications of age-dependent risks of disease and the role of reinfection. Epidemiology and infection. 1997;119(2):183-201.

28. Becerra MC, Appleton SC, Franke MF, Chalco K, Arteaga F, Bayona J et al. Tuberculosis burden in households of patients with multidrug-resistant and extensively drug-resistant tuberculosis: a retrospective cohort study. Lancet. 2011;377(9760):147-52. doi:10.1016/s0140-6736(10)61972-1. 
29. Naidoo CC, Pillay M. Increased in vitro fitness of multi- and extensively drug-resistant

F15/LAM4/KZN strains of Mycobacterium tuberculosis. Clinical microbiology and infection : the official publication of the European Society of Clinical Microbiology and Infectious Diseases. 2014;20(6):O361-9. doi:10.1111/1469-0691.12415.

30. Smith KL, Saini D, Bardarov S, Larsen M, Frothingham R, Gandhi NR et al. Reduced virulence of an extensively drug-resistant outbreak strain of Mycobacterium tuberculosis in a murine model. PloS one.

2014;9(4):e94953. doi:10.1371/journal.pone.0094953. 